\title{
The effect of the Carer Support Needs Assessment Tool intervention (CSNAT-I) \\ in the Danish specialised palliative care setting: a stepped wedge cluster randomised controlled trial
}

By

Line Lund, MSc $\mathrm{PhD}^{\mathrm{a}}$

Lone Ross, MD PhD ${ }^{\mathrm{a}}$

Morten Aagaard Petersen, MSc ${ }^{\mathrm{a}}$

Annemette Blach, $\mathrm{RN}^{\mathrm{b}}$

Elizabeth Rosted, $\mathrm{MScN} \mathrm{PhD}^{\mathrm{c}}$

Georg Bollig, MD MAS PhD ${ }^{\mathrm{d}, \mathrm{e}}$

Gitte Irene Juhl, MD PhD ${ }^{\mathrm{f}}$

Hanne Fahrholt, $\mathrm{RN}^{\mathrm{g}}$

Helen Winther, $\mathrm{MSc}^{\mathrm{h}}$

Louise Laursen, $\mathrm{BScN}^{\mathrm{i}}$

Marianne Hasse, $\mathrm{RN}^{\mathrm{j}}$

Signe Weensgaard, $\mathrm{RN}^{\mathrm{k}}$

Mai-Britt Guldin, MSc $\mathrm{PhD}^{1}$

Gail Ewing, BSc $\mathrm{PhD}^{\mathrm{m}}$

Gunn Grande, BA MPhil PhD ${ }^{\mathrm{n}}$

Mogens Groenvold, MD PhD DMSc ${ }^{\mathrm{a}, \mathrm{o}}$

${ }^{a}$ Palliative Care Research Unit, Department of Geriatrics and Palliative Medicine GP, Bispebjerg and Frederiksberg Hospitals, University of Copenhagen, Copenhagen, Denmark

${ }^{\mathrm{b}}$ Hospice Soendergaard, Maaloev, Denmark

${ }^{c}$ Department of Oncology and Palliative Care, Zealand University Hospital, Roskilde, Denmark

${ }^{\mathrm{d}}$ Palliative Care Team, Medical Department Soenderborg/Toender, South Jutland Hospital, Soenderborg, Denmark

${ }^{\text {e }}$ Medical Research Unit, Institute of Regional Health Research, University of Southern Denmark, Aabenraa, Denmark

${ }^{\mathrm{f}}$ Palliative Care Unit, Department of Oncology and Palliative Care, North Zealand Hospital, Frederikssund, Denmark 
g Department of Geriatrics and Palliative Medicine GP, Bispebjerg and Frederiksberg Hospitals, University of Copenhagen, Copenhagen, Denmark

${ }^{\text {h }}$ Palliative Care Unit, Odense University Hospital, Odense, Denmark.

${ }^{\mathrm{i}}$ Department of Palliative Care, Rigshospitalet - Copenhagen University Hospital, Copenhagen, Denmark.

${ }^{j}$ Diakonissestiftelsens Hospice, Frederiksberg, Denmark

${ }^{\mathrm{k}}$ Arresoedal Hospice, Frederiksvaerk, Denmark

${ }^{1}$ Palliative Care Team, Department of Oncology, Aarhus University Hospital, Aarhus, Denmark

${ }^{\mathrm{m}}$ Centre for Family Research, University of Cambridge, Cambridge, United Kingdom

${ }^{n}$ School of Health Sciences, University of Manchester, Manchester, United Kingdom

${ }^{\circ}$ Section of Health Services Research, Department of Public Health, University of Copenhagen, Copenhagen, Denmark

Corresponding author: Line Lund, Palliative Care Research Unit, Department of Geriatrics and Palliative Medicine GP, Bispebjerg and Frederiksberg Hospitals, University of Copenhagen, Bispebjerg Bakke 23, DK-2400 Copenhagen NV, Denmark. Tel.: +45 232986 33. Fax: +45 3531 20 71. Email: line.lund.01@ regionh.dk

Author contributions: All authors meet the ICMJE requirements for authorship

Data availability statement: Data are available upon reasonable request. Any data sharing requires approval from the Danish Data Protection Board.

Funding: The study was funded by the Danish Cancer Society (Grant A8861) and the A.P. Moeller and Chastine Mc-Kinney Moeller Foundation (Grant 11692). The sponsors had no role in the study design, data collection, analysis and interpretation of data, in the writing of the paper, or in the decision to submit the paper for publication.

Competing Interests: None declared 
Licence for Publication: The Corresponding Author has the right to grant on behalf of all authors and does grant on behalf of all authors, an exclusive licence (or non-exclusive for government employees) on a worldwide basis to the BMJ Publishing Group Ltd to permit this article (if accepted) to be published in BMJ Supportive \& Palliative Care and any other BMJPGL products and sublicences such use and exploit all subsidiary rights, as set out in our licence (http://group.bmj.com /products/journals/instructions-for-authors/licence-forms).

WORD COUNT: 3831 


\section{ABSTRACT}

Background: The Carer Support Needs Assessment Tool intervention (CSNAT-I) has been shown to improve end-of-life care support for informal caregivers. This study investigated the impact of the CSNAT-I on caregivers of patients recently enrolled in specialised palliative care (SPC) at home in Denmark.

Methods: A stepped-wedge cluster randomised controlled trial with nine clusters (i.e., SPC teams). Outcome measures were collected using caregiver questionnaires at baseline (T0) and two (T1) and four (T2) weeks follow-up. ClinicalTrials.gov ID: NCT03466580.

Results: A total of 437 caregivers were enrolled (control group, $n=255$; intervention group, $n=182$ ). No intervention effect was found on the primary outcome, caregiver strain at $\mathrm{T} 1(\mathrm{p}=0.1865)$. However, positive effects were found at T1 and T2 on attention to caregivers' wellbeing $(p<0.0001)$, quality of information and communication $(p<0.0001)$, amount of information $(\mathrm{T} 1$ : $p=0.0002$; T2: $\mathrm{p}<0.0001)$, involvement $(\mathrm{T} 1: \mathrm{p}=0.0045 ; \mathrm{T} 2: \mathrm{p}<0.0001)$, talking about greatest burdens $(\mathrm{p}<0.0001)$ and assistance in managing greatest burdens $(\mathrm{p}<0.0001)$. The effect sizes of these differences were medium or large and seemed to increase from $\mathrm{T} 1$ to $\mathrm{T} 2$. At T1, positive effects were found on distress $(p=0.0178)$ and home care responsibility $(p=0.0024)$. No effect was found on the remaining outcomes.

Conclusion: Although no effect was found on caregiver strain, the CSNAT-I showed positive effects on caregiver distress, home care responsibility and key outcomes regarding caregivers' experience of the interaction with health care professionals. 


\section{INTRODUCTION}

Informal caregivers are central to the support and care of patients,[1;2], particularly when the patient is cared for at home,[3;4]. However, caregivers themselves can be considered 'hidden patients',[5] as they may experience physical, psychological and social morbidity,[6] because the role as caregiver can be overwhelming, go on for years, and may increase towards the end of life, $[7 ; 8]$.

Yet, even in modern specialised palliative care (SPC) where caregiver support is a core function,[9], caregivers do not always feel well supported: they may lack information,[10] and feel overlooked and that their care expertise is not taken into consideration,[11]. In a study amongst Danish patients who were in contact with an SPC team/hospice, a large proportion reported room for improvement in caregiver support, and in the qualitative comments, the patients asked for more support for the family,[12]. Also, in a survey amongst 590 Danish cancer caregivers, a large proportion of caregivers reported lack of support, attention, and information, and insufficient involvement in the patients' disease, treatment and/or care,[13]. Evidence-based interventions and strategies to assess and respond to caregivers' needs have been lacking, $[14 ; 15]$.

The Carer Support Needs Assessment Tool intervention (CSNAT-I) was developed to respond to these problems,[16;17]. The intervention specifically targets caregivers of patients receiving end-oflife care at home and was developed on the basis of qualitative research (focus group and telephone interviews with 75 bereaved caregivers),[16] and validated in 225 caregivers in Hospice Home Care services,[17]. The CSNAT-I is underpinned by a person-centred framework in which the caregiver is taking the lead, and the health care professional (HCP) is working 'with' rather than doing 'to' the caregiver,[18] The CSNAT-I has shown positive effects in studies from Australia and the UK,[1921] and has been valued by both caregivers and HCPs,[22-24].

Whilst the CSNAT-I seems to be a promising, available method to improve support to caregivers of patients in SPC at home in Denmark, evaluation of the intervention in this setting has not been done. Thus, the aim of the present study was to investigate the impact of the CSNAT-I on caregivers of patients who recently started in SPC at home in Denmark: what is the effect of the intervention on caregiver strain, distress, positive caregiving appraisals, experiences of the interaction with HCPs, workload, emotional functioning, fatigue and quality of life? 


\section{METHODS}

\section{Setting}

In Denmark, SPC is delivered as in-patient care at hospices or hospital SPC departments, or as outpatient care from these units in the home or in out-patient clinics. SPC teams are interdisciplinary and comprise specialised medical doctors and nurses and e.g., physiotherapists, social workers, psychologists and priests. Of the 24 SPC teams delivering out-patient care in Denmark (in 2017), nine consented to participate in the present study (Figure 1).

\section{Study design}

The study was a stepped-wedge cluster randomised controlled trial (SW-CRT),[25;26]. In an SWCRT, every cluster first acts as a control and later as an intervention site. The time of transition varies and is determined by randomisation. The SW-CRT design was chosen to account for variation between SPC units, to prevent contamination, and to make participation in the study attractive for SPC units as all units ultimately will deliver the intervention,[25;26].

All participating SPC teams (i.e., clusters) had an enrolment period of 12 months. The shift from preintervention period (control period) to intervention period was at either 3, 6 or 9 months (Figure 1). The randomisation was computer based (carried out by the research group) and allocated three SPC teams to shift to intervention period at each of the three time points.

\section{Study population}

The study population was primary caregivers of patients recently started in SPC delivered by an SPC team. In Denmark, patients receiving SPC are almost exclusively cancer patients. Cancer patients starting in SPC have a very high symptom load (especially fatigue, anorexia and pain), poor level of physical functioning and low quality of life. In 2018 and 2019, the median survival time from start of SPC was 42 and 40 days, respectively,[27;28]

'Primary caregiver' was defined as the lay person whom the patient considered most involved in his/her disease course. Caregivers had to be adults (18 years and over) who were able to read and understand Danish and who were caring for adult patients who were able to read and understand Danish and who had not received any kind of SPC before. Caregivers were not eligible for the study if they had a known cognitive impairment precluding participation or, based on the HCP's clinical judgement, were too distressed to cope with research.

\section{Enrolment procedure}


HCPs briefly introduced caregivers and patients to the study and if they both consented, the research group contacted the caregiver, provided comprehensive information about the study and enrolled him/her in the study. Details of the enrolment procedure are shown in Figure 2.

\section{Intervention}

\section{Content and delivery}

The CSNAT-I has two parts: 1) an evidence-based tool (the CSNAT) consisting of 14 support domains covering the support that caregivers need to a) care for the patient (caregiver as co-worker) and b) look after their own health and well-being (caregiver as client),[16] (Box 1). The tool itself is integrated into 2) a five-stage person-centred framework which is HCP facilitated, but caregiver led (The CSNAT Approach),[18].

Delivery of the CSNAT-I followed the stages of the CSNAT Approach,[18]. First, the CSNAT was introduced to the caregiver by the HCP and/or research group during enrolment and once again when the HCP called the caregiver to plan a CSNAT conversation. Then, the caregiver was given time to consider in which areas he/she needed more support and complete the tool. Subsequently, a CSNAT conversation between the caregiver and the HCP was held, in which the caregiver had the opportunity to identify his/her individual support needs within the highlighted domains and was asked which support needs were the most urgent, what the caregiver felt would help him/her, and whether he/she needed assistance to access the support. The conversation formed the basis for developing a shared action plan which could contain e.g. the caregiver's planned actions to access support or the HCP's planned actions to facilitate support (by e.g. delivering support such as information or comfort, or to refer the caregiver to other services or sources of support such as a social worker or caregiver groups). Finally, a shared review of the caregiver's support needs and action plans was included in a second CSNAT-I.

The CSNAT-I was delivered to each caregiver individually. The CSNAT was either self-completed (preferably) by the caregiver or completed jointly with the HCP. When possible, the subsequent conversation was carried out face-to-face, but phone and video were also allowed. The intervention was delivered by nurses in all SPC teams and additional professionals in some teams. The intervention was delivered twice to each caregiver: first within 13 days of study enrolment and the second 15-27 days after enrolment (Figure 2).

\section{Training}


All HCPs delivering the CSNAT-I received training from the research group (LL and LR). Training consisted of one group training session lasting 2-3 hours provided shortly before the shift to intervention period. The CSNAT training materials can be accessed via http://csnat.org. If needed, refresher training sessions were conducted during the intervention period.

\section{Documentation}

After each intervention, the HCP filled in a standardised documentation form describing the intervention framework (place, duration, etc.).

\section{Data collection and outcome measures}

\section{Caregiver completed standard measures}

Outcome measures were collected at study enrolment (baseline, T0) and at follow-up two (T1) and four (T2) weeks later (Figure 2). These follow-up times were selected to minimize drop-out and to ensure that the intervention was also feasible for caregivers of patients with only a short survival time. The caregiver could choose paper questionnaires, electronic questionnaires, or telephone interviews. If the patient died during the study, subsequent data collection was cancelled.

The primary outcome was caregiver strain at T1 assessed by the eight item strain subscale of the Family Appraisal of Caregiving Questionnaire for Palliative Care (FACQ-PC),[29].

Secondary outcomes were caregiver strain at T2 and the following at T1 and T2:

Experiences of the interaction with HCPs

- Lack of attention from HCPs to caregivers' wellbeing (four item subscale); problems with quality of information from and communication with HCPs (seven item subscale); caregiver involvement (single item); and selected items from the subscale Lack of information from HCPs (six items, reported as one subscale as Cronbach's Alpha: 0.88), all from the Cancer Caregiving Tasks, Consequences and Needs Questionnaire (CaTCoN),[13;30;31]. Minor adjustments were made to some $\mathrm{CaTCoN}$ items to adapt them to the current study (Appendix 1).

- Talking to HCPs about greatest burdens and assistance from HCPs in managing greatest burdens, assessed by two newly developed items.

Experiences of caregiving

- Caregiving workload, assessed by four items (selected from a five item subscale) from the CaTCoN,[13;30;31] 
- Positive caregiving appraisals and caregiver distress, assessed by the respective two subscales (four and seven items, respectively) from the FACQ-PC,[29].

Caregiver wellbeing

- Emotional functioning and fatigue, assessed by subscales consisting of their respective four and three items from the EORTC Quality of Life Questionnaire Core 30 (EORTC QLQC30),[32;32] each supplemented with three selected items from the EORTC Computerized Adaptive Test (EORTC CAT Core) emotional functioning and fatigue item banks, respectively,[33;34], in order to optimize measurement.

- Positive emotional functioning (positive affect), assessed by a subscale (validation not published) of five positively formulated emotional functioning items (originally developed for the EORTC CAT Core emotional functioning item bank, but not included in the final version as they formed a distinct scale).

Caregiver quality of life, assessed by the two-items subscale assessing overall health and quality of life in EORTC QLQ-C30,[32].

For each of the instruments, items and subscales were scored according to usual practice,[13;29;3137]. The FACQ-PC subscale scores were calculated when at least half of the items were completed. The two newly developed items were scored using the CaTCoN scoring procedure,[13].

The questionnaire package (except the positively formulated emotional functioning items which were included later in the process) was evaluated in cognitive interviews with caregivers, ensuring that all items were understood in the context of the present study (Appendix 2).

An overview of the complete caregiver questionnaire is given in Appendix 3.

\section{Caregiver and patient data}

Caregiver socio-demographic data concerning age, gender, relationship to the patient, level of education, employment, and place of residence were collected at baseline (included in the caregiver questionnaire).

Data concerning enrolled patients' diagnosis and SPC course as well as the total number of patients starting SPC at home in the study period in the participating SPC units were extracted from the Danish Palliative Database (DPD). Patient age and gender were provided by the patient when consenting to data extraction.

CSNAT-I data 
Data about delivery of the CSNAT-I were obtained from the HCPs' documentation of the intervention (time, place, duration, whether the patient was present, and the HCP's profession), and all SPC teams were asked to keep a record with the number of caregivers not participating in the study and reasons for non-participation.

\section{Translation}

The CSNAT and FACQ-PC were not available in Danish and were therefore translated following the EORTC Quality of Life Group translation procedure,[38].

\section{Sample size}

This study used the same primary outcome and principles for sample size estimation as the CSNAT study by Aoun,[19]: a moderate effect size of 0.41 for the primary outcome, a power of 0.80 , and an adjustment for cluster effect of 1.62. The required sample size was 308 caregivers (154 in each group). We anticipated that at least $90 \%$ of those who consented would complete baseline measures, and that the drop-out at two weeks follow-up would be $30 \%$ (based on the study by Aoun et al. (2015) which had a mean follow-up time of 43 days and drop-out rate of $45 \%$ ). Based on these assumptions, a total of 490 caregivers had to be enrolled.

\section{Statistical analyses}

All analyses were carried out in SAS Enterprise Guide v.7.11, using a level of significance of 5\%. No imputations for missing data were made.

Distribution of background variables in the intervention and control groups were compared using $\mathrm{Chi}^{2}$ and Wilcoxon rank sum tests.

The effects of the intervention were assessed by comparing differences in change scores from baseline (T0) to follow-up at two (T1) and four weeks (T2) in the intervention and control group, respectively, using a multiple linear regression analysis, adjusting for background variables (shown in Table 1) as well as for baseline score. Effect sizes were calculated using Cohen's d ( i.e., 0.2 small; 0.5 medium; 0.8 large,[39]). Analyses were based on intention to treat. Two sensitivity analyses were carried out: a per protocol analysis (S1) where intervention caregivers who did not receive the CSNAT-I were considered part of the control group, and an analysis excluding intervention caregivers who did not receive the CSNAT-I (S2). Furthermore, a sensitivity analysis investigating the possible confounding effect of (calendar) time was carried out using Wilcoxon rank sum test. 
Table 1. Characteristics (self-reported and obtained from the Danish Palliative Database) of the participating 437 caregivers

\begin{tabular}{llll} 
TOTAL & $\begin{array}{l}\text { INTERVENTION } \\
\text { GROUP } \\
(\mathbf{n}=182)\end{array}$ & $\begin{array}{l}\text { CONTROL } \\
\text { GROUP } \\
(\mathbf{n}=255)\end{array}$ & P-value $^{\mathrm{a}}$ \\
& & & \\
\hline
\end{tabular}

\section{Gender}

Male

Female

Age

Mean

Median

Range

Missing

\section{Relationship to the patient}

Spouse/partner

(Adult) child

Other (e.g., parent, sibling, friend)

Missing

\section{Level of education}

University education

Longer theoretical education (3-4 years)

Short theoretical education (1-3 years)

Other (short education ( $<1$ year), non-theoretical education, ongoing education, no education)

Missing

\section{Employment}

Currently working (full or part time)

Currently on sick leave or has care leave

Retired

Other (student, un-employed, housewife)

Missing

\section{Place of residence}

City or suburbs

Provincial town

Village

Countryside

Missing

\section{Related to patients with}

\section{Gender}

Male

Female

$\begin{array}{lll}135(31 \%) & 55(30 \%) & 80(31 \%) \\ 302(69 \%) & 127(70 \%) & 175(69 \%) \\ & & \\ 59.8 & 57.3 & 61.7 \\ 61.0 & 58.0 & 63.0 \\ 23.0-90.0 & 23.0-84.0 & 24.0-90.0 \\ 23 & 5 & 18\end{array}$

0.7971

0.0009

0. 0010

$\begin{array}{lll}27(64 \%) & 99(57 \%) & 171(70 \%) \\ 125(30 \%) & 69(39 \%) & 56(23 \%) \\ 24(6 \%) & 7(4 \%) & 17(7 \%) \\ 18 & 7 & 11\end{array}$

0.1149

$\begin{array}{lll}60(14 \%) & 33(19 \%) & 27(11 \%) \\ 152(36 \%) & 62(35 \%) & 90(37 \%) \\ 101(24 \%) & 36(20 \%) & 65(26 \%) \\ & & \\ 110(26 \%) & 46(26 \%) & 64(26 \%) \\ 14 & 5 & 9\end{array}$

$\begin{array}{lll}148(34 \%) & 63(35 \%) & 85(34 \%) \\ 92(21 \%) & 43(24 \%) & 49(20 \%) \\ 169(39 \%) & 62(35 \%) & 107(43 \%) \\ 21(5 \%) & 11(6 \%) & 10(4 \%) \\ 7 & 3 & 4\end{array}$

0.2909

$\begin{array}{lll}147(34 \%) & 64(36 \%) & 83(33 \%) \\ 93(22 \%) & 33(18 \%) & 60(24 \%) \\ 98(23 \%) & 37(21 \%) & 61(25 \%) \\ 90(21 \%) & 46(26 \%) & 44(18 \%) \\ 9 & 2 & 7\end{array}$

0.1355

$\begin{array}{lll}218(50 \%) & 82(45 \%) & 136(53 \%) \\ 219(50 \%) & 100(55 \%) & 119(47 \%)\end{array}$

0.0880 
Table 1, continued

Age

Mean

Median

Range

\section{Diagnosis}

Respiratory system cancer

Breast cancer

Pancreas cancer

Colorectal cancer

Prostate

Gynecological cancer

Upper gastrointestinal cancer

Urinary cancer

Cancer in brain and central nerve system

Liver/biliary cancer

Hematological cancer

Head and neck cancer

Other cancer (e.g., melanoma, sarcoma)

Unknown cancer

Not cancer (heart, lung, kidney or

neurological disease)

\section{SPC team from}

Bispebjerg Hospital

Hospice Soendergaard

South Jutland Hospital

Arresoedal Hospice

Diakonissestiftelsens Hospice

North Zealand Hospital

Odense University Hospital \&

Hospital Svendborg

Rigshospitalet

Zealand University Hospital
69.2

71.0

69.5

27.1-96.9

71.8

29.9-90.6

69.0

70.5

27.1-96.9

$93(21 \%)$

$42(10 \%)$

$37(9 \%)$

$38(9 \%)$

$36(8 \%)$

$26(6 \%)$

$28(6 \%)$

$22(5 \%)$

$17(4 \%)$

$16(4 \%)$

$14(3 \%)$

$12(3 \%)$

$23(5 \%)$

$9(2 \%)$

$24(5 \%)$

$41(23 \%)$

$16(9 \%)$

$18(10 \%)$

$16(9 \%)$

$13(7 \%)$

$14(8 \%)$

$11(6 \%)$

$7(4 \%)$

$9(5 \%)$

$9(5 \%)$

$6(3 \%)$

$4(2 \%)$

$10(5 \%)$

$3(2 \%)$

$5(3 \%)$

$52(20 \%)$

$26(10 \%)$

$19(7 \%)$

$22(9 \%)$

$23(9 \%)$

$12(5 \%)$

$17(7 \%)$

$15(6 \%)$

$8(3 \%)$

$7(3 \%)$

$8(3 \%)$

$8(3 \%)$

$13(5 \%)$

$6(2 \%)$

$19(7 \%)$

$56(13 \%)$

$20(5 \%)$

58 (13\%)

$36(20 \%)$

$9(5 \%)$

$20(8 \%)$

$11(4 \%)$

$12(5 \%)$

$24(5 \%)$

$46(25 \%)$

$10(5 \%)$

$12(7 \%)$

$14(5 \%)$

$11(4 \%)$

$61(14 \%)$

$22(12 \%)$

$39(15 \%)$

$95(22 \%)$

$29(16 \%)$

$66(26 \%)$

$30(7 \%)$

$5(3 \%)$

$70(16 \%)$

$13(7 \%)$

$57(22 \%)$
0.5388

0.6550

$<0.0001^{b}$

0.0788

Time from start SPI to study enrolment

(days)

Mean

7.8

8.4

7.4

Median

Range

6.0

6.0

5.5

Missing

$-52.0$

$0.0-52.0$

0

$0.0-49.0$

5

${ }^{\text {a }}$ Chi Square for categorical variables and Wilcoxon for continuous variables

$\mathrm{b}$ That different proportions of caregivers were recruited to either control or intervention group in the different SPC teams was due to and a natural consequence of the study design 


\section{RESULTS}

\section{Study population}

For six SPC teams, the enrolment period was from $15^{\text {th }}$ March, 2018 to $15^{\text {th }}$ April, 2019 (extended by one month to increase sample size in the intervention group) (Figure 1). Start of enrolment in the remaining three teams was delayed due to practical matters and took place from $15^{\text {th }}$ June, 2018 to $15^{\text {th }}$ June, 2019. To increase the sample size in the intervention group (due to slowing of caregiver enrolment in the intervention period), the largest SPC team shifted to the intervention period after six months instead of nine as decided by the randomisation (Figure 1).

A study flow chart (CONSORT diagram) is shown in Figure 3. Consent rates (proportion consenting to participate out of those invited) were $50 \%$ and $34 \%$ in the control and intervention group, respectively. A total of 437 caregivers were enrolled: 255 in the control group; 182 in the intervention group. Ninety-one caregivers were lost to follow-up at T1 (exclusion/attrition rate: $21 \%$ ), and 139 at T2 (exclusion/attrition rate: 32\%), resulting in 346 (T1) and 298 (T2) returned caregiver questionnaires.

Caregivers predominantly chose to complete the baseline questionnaires on paper (87\%) compared to electronically $(12 \%)$ or over the phone $(<1 \%)$ whereas they preferred to complete the follow-up questionnaires electronically $(63 \%)$ followed by on paper $(36 \%)$ and rarely over the phone $(<1 \%)$.

Characteristics of the 437 caregivers are shown in Table 1. Compared to the control group, the intervention group contained significantly more adult children of the patients $(p=0.0010)$ and thus caregivers of younger age $(\mathrm{p}=0.0009)$. No other significant differences between caregivers in the two groups were found.

\section{The intervention}

Of the 142 caregivers in the intervention group completing the follow-up questionnaire at T1, 130 (92\%) had, as intended, received their first CSNAT-I. Of these, four caregivers (3\%) had also received their second CSNAT-I. Twelve caregivers (9\%) had not received a CSNAT-I.

Of the 123 caregivers in the intervention group completing the follow-up questionnaire at $\mathrm{T} 2,93$ (76\%) had, as intended, received two CSNAT-I. Twenty-five caregivers (20\%) had received only one CSNAT-I, and five caregivers (4\%) had still not received a CSNAT-I. 
CSNAT conversations were carried out predominantly by a nurse $(93 \%$ and $91 \%$ of the first and second conversations, respectively) and in the remaining cases by a social worker, priest, psychologist, physiotherapist, medical doctor, or a nurse and a medical doctor together.

Most CSNAT conversations took place in out-patient clinics (37\% and 35\% of the first and second conversations, respectively) followed by the joint home of the patient and caregiver (24\% and $22 \%$ of the first and second conversations, respectively) and over the phone (19\% and $28 \%$ of the first and second conversations, respectively). 'Other places' and information not completed by the HCP totalled $20 \%$ and $15 \%$ of the first and second conversations, respectively.

Ninety-seven percent and $98 \%$ of the first and second conversations, respectively, were conducted with the caregiver alone (i.e., without the patient being present).

The mean duration of the first and second CSNAT conversations was 43 minutes (range 10-90) and 32 (10-70) minutes, respectively.

\section{Effect of the intervention}

No significant effect of the intervention on the primary outcome, caregiver strain at $\mathrm{T} 1$, was found (p $=0.1865)($ Table 2).

However, at T1, positive intervention effects were found on caregiver distress $(\mathrm{p}=0.0178, d=0.12)$, attention to the caregivers' wellbeing ( $\mathrm{p}<0.0001, d=0.84$ ), quality of information and communication ( $\mathrm{p}<0.0001, d=0.57$ ), amount of information $(\mathrm{p}=0.0002, d=0.51)$, responsibility in relation to home care $(\mathrm{p}=0.0024, d=0.17)$, caregiver involvement $(\mathrm{p}=0.0045, d=0.49)$, talking about greatest burdens $(\mathrm{p}<0.0001 ; d=0.98)$ and assistance in managing greatest burdens $(\mathrm{p}<$ $0.0001, d=0.75)$.

At T2, positive intervention effects were found on attention to the caregivers' wellbeing $(\mathrm{p}<0.0001$, $d=0.95)$, quality of information and communication ( $\mathrm{p}<0.0001, d=0.88)$, amount of information ( $\mathrm{p}<0.0001, d=0.73$ ), caregiver involvement ( $<<0.0001, d=0.70)$, talking about greatest burdens $(\mathrm{p}<0.0001, d=1.10)$ and assistance in managing greatest burdens $(\mathrm{p}<0.0001, d=0.84)$.

The two sensitivity analyses (S1 and S2) supported the findings from the main analysis, except that additional, positive intervention effects were seen at T1 on caregiver strain (primary outcome) (S1:p $=0.0048, d=0.28 ; \mathrm{S} 2: \mathrm{p}=0.0288, d=0.23)$ as well as on emotional functioning ( $1: \mathrm{p}=0.0319$, $d=0.19$ ), fatigue ( $\mathrm{S} 1: \mathrm{p}=0.0181, d=0.28 ; \mathrm{S} 2: \mathrm{p}=0.0433, d=0.25$ ) and the caregivers' provision of practical help to the patient $(\mathrm{S} 1: \mathrm{p}=0.0108, d=0.21$ ). 
No confounding effect of (calendar) time was found. 
Table 2. The intervention effect on the primary and secondary outcomes from the Family Appraisal of Caregiving Questionnaire for Palliative Care (FACQ-PC) ${ }^{\text {a }}$, the Cancer

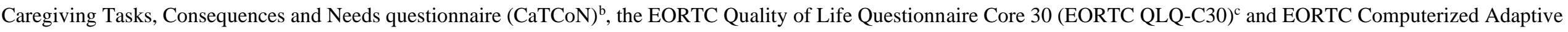
Test (EORTC CAT Core) item banks ${ }^{\mathrm{c}}$, and two newly developed items ${ }^{\mathrm{b}}$ at T1 (two weeks follow-up) and T2 (four weeks follow-up).

\begin{tabular}{|c|c|c|c|c|c|c|c|c|c|c|}
\hline & \multirow[t]{2}{*}{ Time } & \multicolumn{3}{|c|}{ Intervention } & \multicolumn{3}{|c|}{ Control } & \multirow{2}{*}{$\begin{array}{l}\text { Difference } \\
\text { between } \\
\text { change } \\
\text { scores }^{\text {d }}\end{array}$} & \multirow{2}{*}{$\begin{array}{l}\text { Effect size } \\
\text { (Cohen's d) }\end{array}$} & \multirow[t]{2}{*}{$\mathbf{P}^{\mathbf{e}}$} \\
\hline & & $\mathbf{N}$ & $\begin{array}{l}\text { Baseline } \\
\text { mean (SD) }\end{array}$ & $\begin{array}{l}\text { Change from } \\
\text { baseline to } 2 \\
\text { weeks follow- } \\
\text { up (SD) }\end{array}$ & $\mathbf{N}$ & $\begin{array}{l}\text { Baseline } \\
\text { mean (SD) }\end{array}$ & $\begin{array}{l}\text { Change from } \\
\text { baseline to } 2 \\
\text { weeks follow- } \\
\text { up (SD) }\end{array}$ & & & \\
\hline \multicolumn{11}{|l|}{ PRIMARY OUTCOME } \\
\hline \multirow{2}{*}{$\begin{array}{l}\text { Caregiver strain } \\
\text { (FACQ-PC eight item subscale) }\end{array}$} & $\mathrm{T} 1$ & 140 & $2.89(0.78)$ & $-0.02(0.48)$ & 199 & $2.89(0.79)$ & $0.05(0.47)$ & -0.07 & 0.15 & 0.1865 \\
\hline & $\mathrm{T} 2$ & 122 & $2.90(0.74)$ & $-0.04(0.52)$ & 170 & $2.90(0.76)$ & $0.06(0.54)$ & -0.10 & 0.19 & 0.1533 \\
\hline \multicolumn{11}{|l|}{ SECONDARY OUTCOMES } \\
\hline \multicolumn{11}{|l|}{ FACQ-PC } \\
\hline \multirow[t]{2}{*}{ Caregiver distress (four item subscale) } & $\mathrm{T} 1$ & 140 & $2.81(0.95)$ & $-0.10(0.69)$ & 200 & $2.99(0.92)$ & $-0.02(0.70)$ & -0.08 & 0.12 & 0.0178 \\
\hline & $\mathrm{T} 2$ & 122 & $2.80(0.98)$ & $-0.03(0.73)$ & 171 & $2.95(9.96)$ & $0.00(0.73)$ & -0.03 & 0.04 & 0.4424 \\
\hline \multirow[t]{2}{*}{ Positive caregiving appraisals (seven item subscale) } & $\mathrm{T} 1$ & 141 & $4.06(0.57)$ & $-0.08(0.39)$ & 200 & $3.96(0.58)$ & $-0.05(0.41)$ & -0.03 & 0.08 & 0.6792 \\
\hline & $\mathrm{T} 2$ & 123 & $4.08(0.57)$ & $-0.10(0.44)$ & 169 & $3.95(0.58)$ & $-0.05(0.41)$ & -0.05 & 0.12 & 0.4409 \\
\hline \multicolumn{11}{|l|}{ CaTCoN } \\
\hline \multirow{2}{*}{$\begin{array}{l}\text { Lack of attention from health care professionals to the } \\
\text { caregivers' wellbeing (four item subscale) }\end{array}$} & $\mathrm{T} 1$ & 125 & $29.27(24.25)$ & $-11.82(22.81)$ & 173 & $34.20(26.55)$ & $8.03(24.62)$ & -19.85 & 0.84 & $<0.0001$ \\
\hline & $\mathrm{T} 2$ & 110 & $30.13(25.72)$ & $-12.42(27.52)$ & 144 & $34.57(27.63)$ & $14.47(29.21)$ & -26.89 & 0.95 & $<0.0001$ \\
\hline \multirow{2}{*}{$\begin{array}{l}\text { Problems with the quality of information from and } \\
\text { communication with health care professionals (seven } \\
\text { item subscale) }\end{array}$} & $\mathrm{T} 1$ & 121 & $21.55(15.73)$ & $-2.07(15.34)$ & 172 & $23.73(18.63)$ & $7.38(17.50)$ & -9.45 & 0.57 & $<0.0001$ \\
\hline & $\mathrm{T} 2$ & 102 & $20.90(15.90)$ & $-3.17(13.06)$ & 139 & $23.66(19.11)$ & $11.12(18.80)$ & -14.29 & 0.88 & $<0.0001$ \\
\hline \multirow{2}{*}{$\begin{array}{l}\text { Lack of information from health care professionals (six } \\
\text { item (modified) subscale) }\end{array}$} & $\mathrm{T} 1$ & 130 & $42.03(28.79)$ & $-10.11(23.65)$ & 186 & $41.71(28.09)$ & $2.65(25.86)$ & -12.76 & 0.51 & 0.0002 \\
\hline & $\mathrm{T} 2$ & 113 & $41.05(29.00)$ & $-15.05(25.84)$ & 154 & $42.36(28.07)$ & $4.39(27.07)$ & -19.44 & 0.73 & $<0.0001$ \\
\hline \multirow[t]{2}{*}{ Provision of practical help (single item) } & $\mathrm{T} 1$ & 141 & $64.30(32.52)$ & $-6.38(27.86)$ & 193 & $66.67(31.37)$ & $-2.76(25.76)$ & -3.62 & 0.13 & 0.0981 \\
\hline & $\mathrm{T} 2$ & 121 & $63.36(31.74)$ & $-7.16(35.54)$ & 166 & $66.87(30.81)$ & $-5.02(27.86)$ & -2.14 & 0.07 & 0.4277 \\
\hline Provision of personal care (single item) & $\mathrm{T} 1$ & 138 & $25.85(30.41)$ & $-1.69(21.43)$ & 187 & $26.56(31.14)$ & $1.78(25.57)$ & -3.47 & 0.15 & 0.5960 \\
\hline
\end{tabular}




\begin{tabular}{|c|c|c|c|c|c|c|c|c|c|c|}
\hline & $\mathrm{T} 2$ & 120 & $25.56(27.92)$ & $-1.39(27.80)$ & 157 & $23.57(29.30)$ & $4.25(27.66)$ & -5.64 & 0.20 & 0.5419 \\
\hline \multirow[t]{2}{*}{ Provision of psychological support (single item) } & $\mathrm{T} 1$ & 141 & $61.23(32.76)$ & $-5.91(30.42)$ & 190 & $61.23(31.42)$ & $0.18(25.77)$ & -6.09 & 0.22 & 0.1255 \\
\hline & $\mathrm{T} 2$ & 122 & $58.47(32.72)$ & $-5.19(30.91)$ & 163 & $61.15(31.48)$ & $-2.04(32.44)$ & -3.15 & 0.10 & 0.2817 \\
\hline \multirow{2}{*}{$\begin{array}{l}\text { Too much responsibility in relation to home care } \\
\text { (single item) }\end{array}$} & $\mathrm{T} 1$ & 134 & $30.85(33.86)$ & $-4.48(31.07)$ & 188 & $37.94(34.16)$ & $0.89(31.49)$ & -5.37 & 0.17 & 0.0024 \\
\hline & $\mathrm{T} 2$ & 115 & $27.54(31.92)$ & $0.00(32.74)$ & 160 & $37.71(33.25)$ & $2.50(30.27)$ & -2.50 & 0.08 & 0.0902 \\
\hline \multirow[t]{2}{*}{ Caregiver involvement (single item) } & $\mathrm{T} 1$ & 113 & $20.65(26.47)$ & $-3.83(27.37)$ & 151 & $21.85(25.25)$ & $11.70(35.11)$ & -15.53 & 0.49 & 0.0045 \\
\hline & $\mathrm{T} 2$ & 97 & $20.62(27.82)$ & $-6.19(26.06)$ & 123 & $22.22(24.39)$ & $15.45(34.75)$ & -21.64 & 0.70 & $<0.0001$ \\
\hline \multicolumn{11}{|l|}{ EORTC QLQ-C30 } \\
\hline \multirow[t]{2}{*}{ Quality of life (two item subscale) } & $\mathrm{T} 1$ & 141 & $47.08(8.83)$ & $-0.64(6.44)$ & 203 & $47.47(8.53)$ & $-0.46(7.48)$ & -0.18 & 0.03 & 0.9274 \\
\hline & $\mathrm{T} 2$ & 122 & $47.12(8.61)$ & $-0.57(7.63)$ & 174 & $47.96(8.25)$ & $-1.50(8.97)$ & 0.93 & 0.11 & 0.7534 \\
\hline \multicolumn{11}{|l|}{ EORTC QLQ-C30 + EORTC CAT Core } \\
\hline \multirow{2}{*}{$\begin{array}{l}\text { Emotional functioning, short-form (seven item } \\
\text { subscale) }\end{array}$} & $\mathrm{T} 1$ & 137 & $42.79(7.78)$ & $1.07(6.01)$ & 193 & $42.47(8.41)$ & $0.40(6.32)$ & 0.67 & 0.11 & 0.2446 \\
\hline & $\mathrm{T} 2$ & 118 & $43.71(8.06)$ & $1.24(7.53)$ & 166 & $42.15(8.75)$ & $0.82(6.58)$ & 0.42 & 0.06 & 0.6286 \\
\hline \multirow[t]{2}{*}{ Fatigue, short-form (six item subscale) } & $\mathrm{T} 1$ & 137 & $56.55(9.48)$ & $-0.05(6.36)$ & 194 & $55.41(9.62)$ & $0.84(6.55)$ & -0.89 & 0.14 & 0.1796 \\
\hline & $\mathrm{T} 2$ & 116 & $55.79(9.44)$ & $0.19(7.36)$ & 164 & $55.36(9.47)$ & $0.79(6.80)$ & -0.60 & 0.08 & 0.7799 \\
\hline \multicolumn{11}{|l|}{ EORTC CAT Core (excluded items) } \\
\hline \multirow[t]{2}{*}{ Positive emotional functioning (five item subscale) } & $\mathrm{T} 1$ & 141 & $45.55(6.96)$ & $-0.32(5.37)$ & 201 & $45.29(6.86)$ & $-0.41(4.88)$ & 0.09 & 0.02 & 0.6964 \\
\hline & $\mathrm{T} 2$ & 121 & $45.71(6.83)$ & $-0.50(5.38)$ & 172 & $45.60(6.88)$ & $-0.20(4.80)$ & -0.30 & 0.06 & 0.6329 \\
\hline \multicolumn{11}{|l|}{ Newly developed items } \\
\hline \multirow[t]{2}{*}{ Talking about greatest burdens (single item) } & $\mathrm{T} 1$ & 95 & $60.35(32.72)$ & $-34.04(38.59)$ & 122 & $63.39(34.66)$ & $0.82(32.49)$ & -34.86 & 0.98 & $<0.0001$ \\
\hline & $\mathrm{T} 2$ & 83 & $60.24(34.32)$ & $-38.96(40.26)$ & 109 & $67.28(31.09)$ & $1.83(33.59)$ & -40.79 & 1.10 & $<0.0001$ \\
\hline \multirow[t]{2}{*}{ Assistance in managing greatest burdens (single item) } & $\mathrm{T} 1$ & 80 & $56.25(32.52)$ & $-24.17(32.68)$ & 104 & $59.62(36.18)$ & $0.64(33.16)$ & -24.81 & 0.75 & $<0.0001$ \\
\hline & $\mathrm{T} 2$ & 70 & $56.19(35.23)$ & $-29.52(40.74)$ & 90 & $60.37(33.48)$ & $3.70(38.53)$ & -33.22 & 0.84 & $<0.0001$ \\
\hline
\end{tabular}

In the analyses, diagnosis was collapsed into eight groups (as opposed to 15 as shown in Table 1)

SD: Standard deviation

${ }^{a}$ Expressed on a scale of 1 (no strain/distress/positive caregiving appraisals) to 5 (maximum strain/distress/positive caregiving appraisals). Subscales are shown in Appendix 3.

${ }^{b}$ Expressed on a scale of 0 (no problems/unmet needs/tasks) to 100 (maximum problems/unmet needs/tasks). Items and subscales are shown in Appendix 3.

${ }^{c}$ Scores are transformed to T-scores, i.e., scored so the European general population has mean=50 and SD=10. The lower the score, the lower the level of quality of life, fatigue, emotional

functioning, and positive emotional functioning. Subscales are shown in Appendix 3.

${ }^{\mathrm{d}}$ For the outcomes 'Positive caregiving appraisals', 'Quality of life', 'Emotional functioning', and 'Positive emotional functioning', a positive value for the 'Difference between change scores in intervention and control groups' indicates that the intervention group had a more favourable development from baseline to follow-up than the control group. For all other outcomes, a negative value for the 'Difference between change scores in intervention and control groups' indicates that the intervention group had a more favourable development from baseline to follow-up than the control group. 
${ }^{e}$ All background variables shown in Table 1 and baseline score for the particular outcome were included in the multiple linear regression (non-reduced model) 


\section{DISCUSSION}

We found no effect of the CSNAT-I on the primary outcome, caregiver strain at T1, in the primary analysis. However, positive intervention effects were found at both T1 and T2 on the caregivers' evaluation of attention to their wellbeing, quality of information and communication, amount of information, caregiver involvement, talking about greatest burdens and assistance in managing greatest burdens. For all of these outcomes, the effect sizes were found to be medium or large and seemed to increase from T1 to T2 (Table 2). Furthermore, at T1, positive effects were found on distress and home care responsibility.

Lack of an intervention effect on the primary outcome may be due to increased caregiver strain being an inevitable consequence of providing end-of-life care for a loved one. However, it should be noted that a reduction in caregiver strain was found at $\mathrm{T} 1$ in both sensitivity analyses, and this may indicate some intervention effect. Furthermore, the positive intervention effect $(d=0.35)$ after two CSNAT-Is reported by Aoun et al. (2015) was based on a per protocol analysis (i.e., as our sensitivity analysis S1), not on intention to treat analysis.

Caregiver strain was chosen as the primary outcome as we wished to replicate the study by Aoun et al (2015), but in addition we included several secondary outcomes which we consider highly relevant and important for the caregivers. The fact that the CSNAT-I had positive effects on caregiver distress, home care responsibility and a wide range of aspects of caregiver-HCP interaction is very encouraging.

It is noteworthy that positive effects were obtained at T1, i.e. after one CSNAT conversation only, that is, an effect obtained quickly and with minimal intervention. This suggests that caregivers of patients with a very limited life expectancy have the potential to benefit from the intervention. Furthermore, as the effect sizes seemed to increase at T2, i.e. after two CSNAT conversations, a doseresponse relationship is suggested, and further research could investigate the impact of additional interventions.

Baseline levels of caregiver strain in the current study $(2.89 ; 2.90)$ and in Aoun et al.'s (2015) study (2.92 in both groups) were very similar. However, it appears that a substantial proportion of caregivers were not informed about our project as HCPs judged the patient or caregiver too burdened for the caregiver to participate (Figure 3). This 'gate keeping' may have excluded some caregivers with a high level of caregiver strain (and/or other problems) and thereby a pronounced need for support from participation. 
Our consent rates of $50 \%$ and $34 \%$ in the control and intervention group, respectively, indicate that compared to agreeing to complete standard measures only (i.e., in the control period), some caregivers may have perceived the CSNAT-I as time consuming and as an additional obligation they preferred to avoid. Therefore, when using the CSNAT-I in routine practice it should be introduced in a way that does not make caregivers feel that 'this is an extra thing to deal with' but rather as an opportunity to have a conversation about their support needs.

A limitation of the study is that the SW-CRT design was not optimal due to differences in the sizes of SPC teams and the slower enrolment rate during the intervention period, and thus minor modifications to the study design were required to ensure sufficient statistical power in analyses. Another limitation is that randomisation was skewed regarding caregiver age and relationship to the patient. However, the study has significant strengths: it is the first multi-centre RCT investigating the effect of the CSNAT-I, and for a palliative care study where enrolment is a known difficulty it has a large sample size (i.e., 36\% more caregivers than in the study by Aoun (2015)) and a low exclusion/attrition rate (32\% at T2 vs. $45 \%$ in the study by Aoun et al (2015)).

There are several areas of further research indicated by the present study. It would be highly relevant to investigate the Danish HCPs' experiences of delivering the CSNAT-I and whether the intervention also has positive effects on the patients (e.g. on their acute hospitalizations, survival time and place of death). Positive HCP experiences and patient effects would further strengthen the case for implementation of the CSNAT-I as standard care in daily clinical practice.

\section{CONCLUSIONS}

This study of the CSNAT-I in the Danish SPC setting found no effect of the intervention on the primary outcome, caregiver strain at two weeks follow-up, in the primary analysis. Yet, positive intervention effects were found on caregiver distress and home care responsibility at two weeks follow-up and on several key outcomes regarding caregivers' experience of the interaction with the HCPs at both two and four weeks follow-up, suggesting that the CSNAT-I is of great value to caregivers.

\section{Compliance with Ethical standards}


The study was carried out in accordance with the 1964 Helsinki Declaration and its later amendments. The study was registered at ClinicalTrials.gov (ID: NCT03466580) and was approved by the Danish Data Protection Board (VD-2018-46). The protocol was presented to the Scientific Ethical Committee system (no. H-16042063) and was found not to require formal approval from the committee.

Informed consent: Patient and caregiver gave informed consent to the caregiver's participation in the study. Furthermore, the patients gave informed consent to extraction of data concerning their diagnosis and SPC course from Danish registers.

\section{Acknowledgements}

The authors wish to thank the participating caregivers and the involved staff.

The CSNAT is a copyright tool which requires a licence for its use. For details about accessing the CSNAT and the licensing process, please visit http://csnat.org, or contact Dr Gail Ewing (ge200@cam.ac.uk) or Professor Gunn Grande (gunn.grande@manchester.ac.uk). 


\section{FIGURE LEGENDS}

Figure 1: The stepped wedge trial design of the study. The planned enrolment period for all participating SPC teams (i.e., clusters) was 12 months. The shift from control period to intervention period was after either 3 (period 1), 6 (period 2) or 9 (period 3) months. For six SPC teams, the enrolment period was extended by one month.

Figure 2: Study course

Figure 3: Study flow chart (CONSORT diagram) 


\section{References}

1. Van Ryn M, Sanders S, Kahn K, et al. Objective burden, resources, and other stressors among informal cancer caregivers: a hidden quality issue? Psychooncology 2011;20:44-52.

2. Danish Health Authority. National recommendations for health care professionals' meeting with caregivers of seriously ill people [In Danish]. 2012.

3. Gomes B, Higginson I. Factors influencing death at home in terminally ill patients with cancer: systematic review. BMJ 2006;332(7540):515-521.

4. Grande G, Ewing G. Death at home unlikely if informal carers prefer otherwise: implications for policy. Palliat Med 2008;22(8):971-972.

5. Kristjanson L, Aoun S. Palliative care for families: remembering the hidden patients. Can $J$ Psychiatry 2004;49(6):359-365.

6. Girgis A, Lambert S, Johnson C, et al. Physical, psychosocial, relationship, and economic burden of caring for people with cancer: a review. J Oncol Pract 2013;9(4):197-202.

7. Zaider T, Kissane D. The assessment and management of family distress during palliative care. Curr Opin Support Palliat Care 2009;3(1):67-71.

8. Williams A, McCorkle R. Cancer family caregivers during the palliative, hospice, and bereavement phases: a review of the descriptive psychosocial literature. Palliat Support Care 2011;9(3):315-325.

9. Sepulveda C, Marlin A, Yoshida T, et al. Palliative care: the World Health Organization's global perspective. J Pain Symptom Manage 2002;24(2):91-96.

10. Wang T, Molassiotis A, Chung B, et al. Unmet care needs of advanced cancer patients and their informal caregivers: a systematic review. BMC Palliat Care 2018;17(1):96.

11. Harding R, Epiphaniou E, Hamilton D, et al. What are the perceived needs and challenges of informal caregivers in home cancer palliative care? Qualitative data to construct a feasible psycho-educational intervention. Support Care Cancer 2012;20(9):1975-1982.

12. Korngut S, Johnsen AT, Spielmann M, et al. Cancer patients' experiences in the palliative phase of the disease course. A barometer survey [In Danish]. The Danish Cancer Society 2012.

13. Lund L, Ross L, Petersen MA, et al. The interaction between informal cancer caregivers and health care professionals: a survey of caregivers' experiences of problems and unmet needs. Support Care Cancer 2015;23:1719-1733.

14. Hudson P, Trauer T, Graham S, et al. A systematic review of instruments related to family caregivers of palliative care patients. Palliat Med 2010;24(7):656-668. 
15. Stajduhar K, Funk L, Toye C, et al. Part 1: Home-based family caregiving at the end of life: a comprehensive review of published quantitative research (1998-2008). Palliat Med 2010;24(6):573-593.

16. Ewing G, Grande G. Development of a Carer Support Needs Assessment Tool (CSNAT) for end-of-life practice at home: A qualitative study. Palliat Med 2013;27(3):244-256.

17. Ewing G, Payne S, Grande G. The Carer Support Needs Assessment Tool (CSNAT) for Use in Palliative and End-of-life Care at Home: A Validation Study. J Pain Symptom Manage 2013;46(3):395-405.

18. Ewing G, Austin L, Diffin J, et al. Developing a person-centred approach to carer assessment and support. Br J Community Nurs 2015;20(12):580-584.

19. Aoun S, Grande G, Howting D, et al. The Impact of the Carer Support Needs Assessment Tool (CSNAT) in Community Palliative Care Using a Stepped Wedge Cluster Trial. PLoS ONE 2015;10(4):e0123012.

20. Grande G, Austin L, Ewing G, et al. Assessing the impact of a Carer Support Needs Assessment Tool (CSNAT) intervention in palliative home care: a stepped wedge cluster trial. BMJ Support Palliat Care 2015;0:1-9.

21. Aoun S, Ewing G, Grande G, et al. The Impact of Supporting Family Caregivers Before Bereavement on Outcomes After Bereavement: Adequacy of End-of-Life Support and Achievement of Preferred Place of Death. J Pain Symptom Manage 2018;55(2):368-378.

22. Aoun S, Toye C, Deas K, et al. Enabling a family caregiver-led assessment of support needs in home-based palliative care: Potential translation into practice. Palliat Med 2015;29(10):929938.

23. Ewing G, Austin L, Grande G. The role of the Carer Support Needs Assessment Tool in palliative home care: A qualitative study of practitioners' perspectives of its impact and mechanisms of action. Palliat Med 2016;30(4):392-400.

24. Aoun S, Deas K, Toye C, et al. Supporting family caregivers to identify their own needs in endof-life care: Qualitative findings from a stepped wedge cluster trial. Palliat Med 2015;29(6):508-517.

25. Mdege N, Man M, Taylor Nee Brown C, et al. Systematic review of stepped wedge cluster randomized trials shows that design is particularly used to evaluate interventions during routine implementation. J Clin Epidemiol 2011;64(9):936-948.

26. Beard E, Lewis J, Copas A, et al. Stepped wedge randomised controlled trials: systematic review of studies published between 2010 and 2014. Trials 2015;16:353.

27. Hansen M, Adsersen M, Groenvold M. Danish Palliative Database: Annual Report 2018 [In Danish]. DMCG-PAL 2019.

28. Hansen M, Adsersen M, Groenvold M. Danish Palliative Database: Annual Report 2019 [In Danish]. DMCG-PAL. 2020. 
29. Cooper B, Kinsella G, Picton C. Development and initial validation of a family appraisal of caregiving questionnaire for palliative care. Psychooncology 2006;15:613-622.

30. Lund L, Ross L, Groenvold M. The initial development of the 'Cancer Caregivng Tasks, Consequences and Needs Questionnaire' (CaTCoN). Acta Oncolog 2012;51(8):1009-1019.

31. Lund L, Ross L, Petersen MA, et al. The validity and reliability of the 'Cancer Caregiving Tasks, Consequences and Needs Questionnaire' (CaTCoN). Acta Oncolog 2014;53(7):966-974.

32. Aaronson N, Ahmedzai S, Bergman B, et al. The European Organisation of Research and Treatment of Cancer QLQ-C30: a quality-of-life instrument for use in international clinical trials in oncology. J Natl Cancer Inst 1993;85(5):365-376.

33. Petersen M, Gamper E, Costantini A, et al. An emotional functioning item bank of 24 items for computerized adaptive testing (CAT) was established. J Clin Epidemiol 2016;70:90-100.

34. Petersen M, Giesinger J, Holzner B, et al. Psychometric evaluation of the EORTC computerized adaptive test (CAT) fatigue item pool. Qual Life Res 2013;22(9):2443-2454.

35. Lund L, Ross L, Petersen MA, et al. Cancer caregiving tasks and consequences and their associations with caregiver status and the caregiver's relationship to the patient: a survey. $B M C$ Cancer 2014;14:541.

36. Petersen M, Aaronson N, Arraras J, et al. The EORTC CAT Core - The computer adaptive version of the EORTC QLQ-C30 questionnaire. Eur J Cancer 2018;100:8-16.

37. Liegl G, Petersen M, Groenvold M, et al. Establishing the European Norm for the health-related quality of life domains of the computer-adaptive test EORTC CAT Core. Eur J Cancer 2019;107:133-141.

38. Dewolf L, Koller M, Velikova G, et al. EORTC Quality of Life Group translation procedure. 3 ed. Brussels, Belgium: EORTC Quality of Life Group 2009.

39. Cohen J. Statistical Power Analysis for the Behavioral Sciences. 2nd ed. New Jersey: Lawrence Erlbaum Associates, Inc. 1988. 